\title{
Mck2-dependent infection of alveolar macrophages promotes replication of MCMV in nodular inflammatory foci of the neonatal lung
}

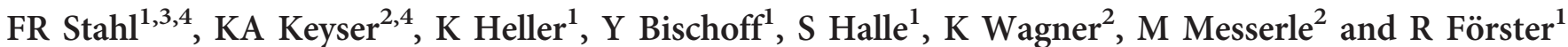

Infection with cytomegalovirus (CMV) shows a worldwide high prevalence with only immunocompromised individuals or newborns to become symptomatic. The host's constitution and the pathogen's virulence determine whether disease occurs after infection. Mouse CMV (MCMV) is an appreciated pathogen for in vivo investigation of host-pathogen interactions. It has recently been reported that a single base pair deletion can spontaneously occur in the open reading frame of MCMV-encoded chemokine 2 (MCK2), preventing the expression of the full-length gene product. To study the consequences of this mutation, we compared the Mck2-defective reporter virus MCMV-3D with the newly generated repaired $\mathrm{Mck2}^{+}$mutant MCMV-3DR. Compared with MCMV-3D, neonatal mice infected with MCMV-3DR showed severe viral disease after lung infection. Viral disease coincided with high viral activity in multiple organs and increased virus replication in previously described nodular inflammatory foci (NIF) in the lung. Notably, MCMV-3DR showed tropism for alveolar macrophages in vitro and in vivo, whereas MCMV-3D did not infect this cell type. Moreover, in vivo depletion of alveolar macrophages reduced MCMV-3DR replication in the lung. We proposed an Mck2-mediated mechanism by which MCMV exploits alveolar macrophages to increase replication upon first encounter with the host's lung mucosa.

\section{INTRODUCTION}

Pathogenesis of infection is determined by dose and site of entry of the causative organism, the pathogen's virulence, and the host's immune response. ${ }^{1}$ Studying the murine cytomegalovirus (MCMV) as a natural mouse pathogen has revealed many aspects of host-pathogen interactions on the cellular, molecular, and genetic level. ${ }^{2-4} \mathrm{MCMV}$ causes disease in mice that are either exposed to high viral doses, have an artificially diminished immune response (knockout mice or mice antibody-depleted of specific immune cells), or are still in their first days of life. ${ }^{3,5-9}$ Viral genetics, especially mutagenesis of the bacterial artificial chromosome (BAC)-cloned MCMV genome, ${ }^{10,11}$ has helped to unmask a series of different virulence factors of MCMV that interfere with immune responses. ${ }^{2,8,12,13}$

The MCMV open reading frame (ORF) m131/m129 codes for the 'MCMV-encoded chemokine 2' (MCK2), which shows sequence similarity to mammalian CC chemokines. ${ }^{14-16}$ This chemokine has been shown to be released from infected cells ${ }^{15}$ and arguably is a transmembrane protein. ${ }^{15,16}$ The role of MCK2 for viral replication has been investigated in vitro and in vivo with the use of various MCMV mutants containing a disruption in the Mck2 ORF. ${ }^{16,17}$ Whereas in vitro replication on murine embryonic fibroblasts is not affected by the absence of $M c k 2$, in vivo viral properties seem to be altered. ${ }^{16-18} M c k 2$ has been shown to be essential for MCMV to establish high viral titers in the salivary gland after infection of adult $\mathrm{BALB} / \mathrm{c}$ mice, while viral replication in other organs seems to be variably affected. ${ }^{16,18-20}$ Furthermore, MCK2 has been proposed to attract inflammatory myeloid progenitor cells to the site of infection to promote monocyte-associated dissemination of MCMV within the host. ${ }^{17,18,21}$ However, in a recent study a role of $M c k 2$ for monocyte recruitment to the spleen has been questioned. ${ }^{22}$ The recruitment of monocytes has additionally

\footnotetext{
${ }^{1}$ Institute of Immunology, Hannover Medical School, Hannover, Germany and ${ }^{2}$ Institute of Virology, Hannover Medical School, Hannover, Germany. Correspondence: FR Stahl or R Förster (f.stahl@uke.de or foerster.reinhold@mh-hannover.de)

${ }^{3}$ Current address: Institute for Clinical Chemistry and Laboratory Medicine, University Medical Center Hamburg-Eppendorf, 20246 Hamburg, Germany.

${ }^{4}$ The first two authors contributed equally to this work.
} 
been implicated in interfering with $\mathrm{CD}^{+} \mathrm{T}$-cell immune response. $^{20,23}$ Thus, $M c k 2$ is a well-established pathogenicity factor of MCMV, which, although not threatening the immunocompetent host's life, promotes viral dissemination to the salivary gland, the virus' favorite niche and important reservoir for transmission.

The first BAC cloning of the MCMV Smith strain led to the generation of the BAC pSM3fr. ${ }^{10,24} \mathrm{~A}$ previous study that focused on DNA sequence analysis of the pSM3fr BAC clone reported on a single base pair deletion positioned in the $m 129$ ORF. ${ }^{19}$ This frameshift mutation is predicted to disrupt $\mathrm{m} 131 /$ m129 translation and therefore would lead to a truncated MCK2 protein. ${ }^{19}$ Comparison of a virus mutant with a restored Mck2 ORF and a mutant derived from the deletion-containing pSM3fr BAC clone revealed lower viral titers in the salivary glands of adult mice infected with the latter mutant, whereas in vitro growth of the two viruses was comparable. These findings are in line with reports by others on $M c k 2^{-} \mathrm{MCMV}$ mutants. ${ }^{15-17}$ Conclusively, the observed attenuation of the pSM3fr-derived virus in salivary glands is due to a mutation in the Mck2 ORF.

We have previously reported on the generation of the reporter virus MCMV-3D that is based on the pSM3fr BAC clone. ${ }^{25}$ Studying MCMV-3D in vivo, we found that neonatal mice cope with infection of this virus in the lung. ${ }^{9}$ Here, we analyzed the DNA sequence of the Mck2 ORF and found the same base pair deletion in MCMV-3D as described by Jordan et al. ${ }^{19}$ Insertion of the missing base pair into the $M c k 2$ ORF led to the generation of the $\mathrm{Mck}^{+}$reporter mutant MCMV-3DR. Laryngopharyngeal infection of neonatal mice with MCMV3DR revealed marked differences in pathogenicity when compared to the unrepaired MCMV-3D. The $\mathrm{Mck2}^{+}$virus interfered with body-weight gain and replicated more efficiently in previously described nodular inflammatory foci (NIF) of the neonatal lung. This was associated with pronounced viral activity not only in the salivary glands but also in all organs of the young mice. Strikingly, comparing the two virus mutants, we observed a different primary viral cell tropism. Whereas MCMV-3D primarily infected lung epithelial cells, MCMV-3DR additionally replicated in alveolar macrophages. Notably, depletion of alveolar macrophages in vivo interfered with the boosted replication of MCMV-3DR. Thus, we revealed a previously unknown feature of $M c k 2$ that allows MCMV to use alveolar macrophages for increased viral replication in the lung mucosa.

\section{RESULTS \\ Mck2 determines MCMV pathogenicity after infection of neonatal mice}

DNA sequencing of the $m 129$ ORF of MCMV-3D revealed the same AT base pair deletion at position 187,786 in the MCMV3D genome when compared with the wild-type Smith strain as described by Jordan et al. ${ }^{19}$ for the BAC pSM3fr. We restored the Mck2 ORF by insertion of the missing base pair and generated a new virus mutant, MCMV-3DR (Figure 1a). MCMV-3D and MCMV-3DR showed similar growth in murine embryonic fibroblasts (Figure 1b). We investigated these two mutants in our recently established model of lung infection in neonatal mice. ${ }^{9}$ Accordingly, we laryngopharyngeally infected neonates with either $5 \times 10^{4}$ plaque-forming units (PFU) MCMV-3D or MCMV-3DR and monitored their health status over time. Animals infected with MCMV-3DR gained less body weight than MCMV-3D-infected neonates or even stopped growing from $\sim 1$ week after infection (Figure 1c). For ethical reasons, we killed neonates fulfilling defined experimental termination criteria (see the Methods section). Therefore, investigation of approximately $80 \%$ MCMV-3DR-infected neonates had to be terminated, mostly around 9-12 days post infection (dpi) (Figure 1d).

Both virus mutants encode Gaussia luciferase, allowing to estimate viral activity in infected organs. ${ }^{25}$ We investigated virally expressed luciferase activity in organs of neonatal mice at different time points after laryngopharyngeal infection. Viral activity of MCMV-3DR was comparable to MCMV-3D in the lung at $1 \mathrm{dpi}$ and absent in all other organs analyzed at this time point (Figure 1e). On the following days, MCMV-3DR replicated to high titers in the respiratory tract and pronounced viral activity in all organs examined was observed, which was significantly higher than in MCMV-3D-infected neonates (Figure 1e). Mice surviving MCMV-3DR infection cleared the virus within 3 weeks in all organs except the salivary glands. Here, luciferase expression measured at 28 dpi seemed to build a plateau on a high level, whereas in salivary glands of MCMV3D-infected mice viral activity did not seem to further increase after $8 \mathrm{dpi}$ and luciferase activity persisted at an approximately two orders of magnitude lower level (Figure 1e). These data suggest that augmented viral replication caused the growth retardation of MCMV-3DR-infected neonates. Interestingly, whereas the levels of viral activity in all organs differed between MCMV-3D- and MCMV-3DR-infected neonates, the spatiotemporal dynamics and clearance of infection in most organs at $\sim 3$ weeks after infection were similar in both groups.

Although MCK2 has been suggested to be a pathogenicity factor in adult mice, the morbidity observed in neonates after infection with MCMV-3DR was rather unexpected. Thus, we intranasally infected adult mice with $10^{6} \mathrm{PFU}$ MCMV-3D and MCMV-3DR to compare virus activity at $8 \mathrm{dpi}$, a time when MCMV replication peaked in neonatal mice. We found MCMV-3DR replication to be increased in lung, salivary glands, and brain, whereas in the spleen, liver, and kidney, the activity of both viruses was low and in similar range (Supplementary Figure 1a online). These data confirm the role of MCK2 in viral dissemination to salivary glands and the increased viral activity of MCMV-3DR at the site of primary infection as observed in neonates. However, adult mice showed no complete systemic infection and no obvious signs of disease within 8 days after MCMV inoculation, suggesting that MCMV-mediated disease is a unique feature in neonatal mice. In summary, whereas differences of in vitro replication characteristics between MCMV-3D and MCMV-3DR seemed at most to be marginal, MCMV-3DR was highly pathogenic in neonates in vivo when compared with MCMV-3D. 

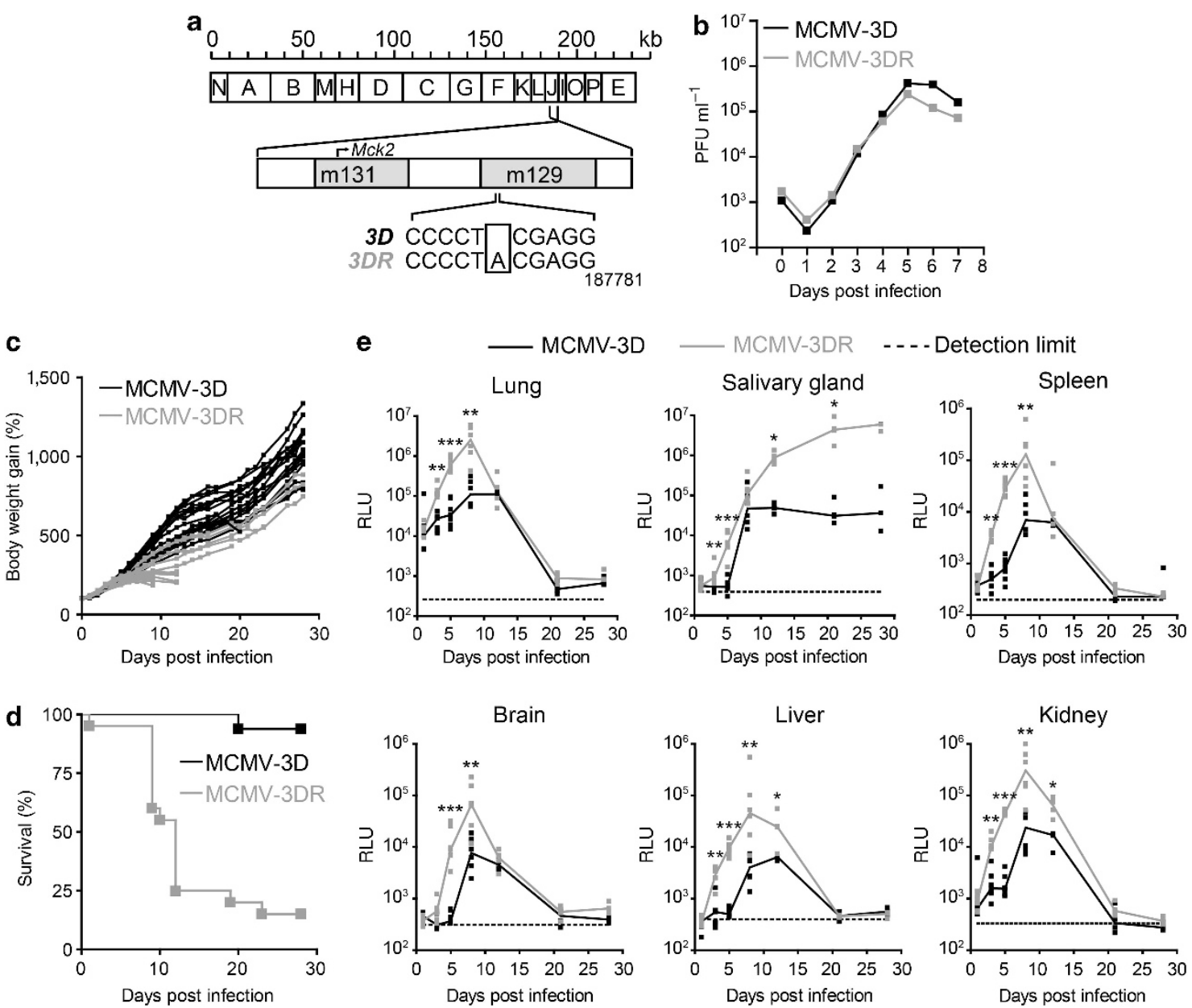

Figure 1 Restoration of the Mck2 open reading frame (ORF) changes mouse cytomegalovirus (MCMV) pathogenicity. (a) Schematic representation of the prototypic orientation of the MCMV genome, with the m131 and m129 ORF depicted below (in reverse orientation). MCMV-3D carries the same deletion of an AT base pair at position 187,786 disrupting the Mck2 ORF as described. ${ }^{19}$ Reinsertion of this base pair allows full-length MCK2 synthesis in MCMV-3DR. (b) Growth of MCMV mutants on murine embryonic fibroblasts upon infection with a multiplicity of infection (MOI) of 0.1. The titers of infectious virus in cell culture supernatants at the designated days post infection were determined by plaque assay with data points representing the mean of triplicate cultures. A representative result of two independent experiments is shown. (c-e) Neonatal mice were laryngopharyngeally infected with $5 \times 10^{4}$ PFUs MCMV mutants as indicated and (c) monitored for body weight gain. (d) Survival curve after MCMV infection. Animals fulfilling the termination criteria were killed. Data were obtained from four independent experiments with $n=16$ (MCMV-3D) and $n=20$ (MCMV-3DR). (e) Viral activity in neonatal organs estimated by the measurement of luciferase activity at time points after infection as indicated. Each value represents one animal, connecting line between medians. Data from MCMV-3D-infected neonates (lung) were in part already published in Stahl et al. ${ }^{9}$ MCK2, MCMV-encoded chemokine 2; PFU, plaque-forming units; RLU, relative light units. Statistical significance depicted as follows: ${ }^{\star} P<0.05 ;{ }^{\star \star} P<0.01$; and ${ }^{\star \star \star} P<0.001$.

\section{Increased viral replication in NIFs in the lung of MCMV-3DR-} infected neonates

NIFs of the neonatal lung are sites of ongoing MCMV replication, which, in addition to infected cells, consist mainly of $\mathrm{CD}^{+} 5^{+}$myeloid cells and also of lymphocytes. ${ }^{9}$ We investigated the presence of NIFs in neonates at 5 dpi with either MCMV-3D or MCMV-3DR (Figure 2a). Both virus mutants encode the fluorescent protein mCherry to allow detection of infected cells. ${ }^{25} \mathrm{We}$ found significantly more NIFs to be present in the left lung lobe in MCMV-3DR-infected mice (Figure 2b,c). High magnification analysis of NIFs revealed notable differences in MCMV-3D-infected neonates when compared to MCMV-3DR-infected neonates (Figure 2d). First, whereas both MCMV-3D and MCMV-3DR infected fibroblast-like stroma cells within NIFs, we additionally found
$\mathrm{CD} 1 \mathrm{~b}^{+} \mathrm{mCherry}^{+}$cells in NIFs of MCMV-3DR-infected neonates, suggesting the infection of myeloid cells (Figure 2e). Second, the size of NIFs in MCMV-3DR-infected neonates was significantly increased (Figure 2f). Third, the number of infected cells per NIF and the number of infected cells per inflammation area was higher in MCMV-3DR-infected neonates (Figure 2g,h). Thus, MCMV-3DR stimulated the formation of NIFs, showed a different cell tropism within NIFs, and the density of infected cells within NIFs was increased after infection with this mutant.

\section{Mck2 mediates infection of alveolar macrophages}

Owing to the striking differences between MCMV-3D and MCMV-3DR in vivo observed at $5 \mathrm{dpi}$, we decided to investigate which cells were primarily infected at $1 \mathrm{dpi}$. We 
a

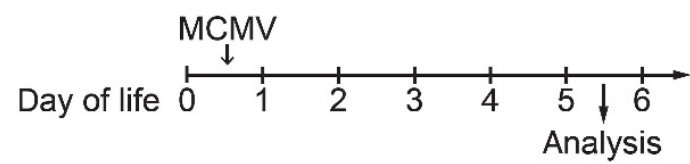

b

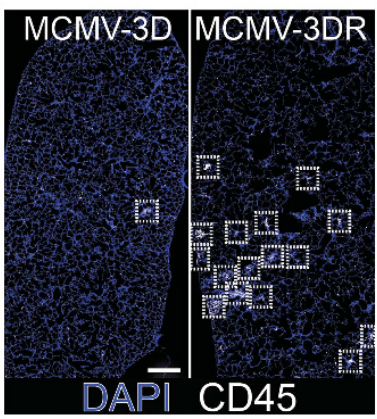

C

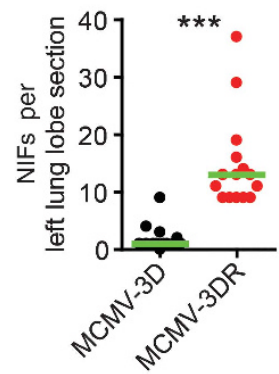

d

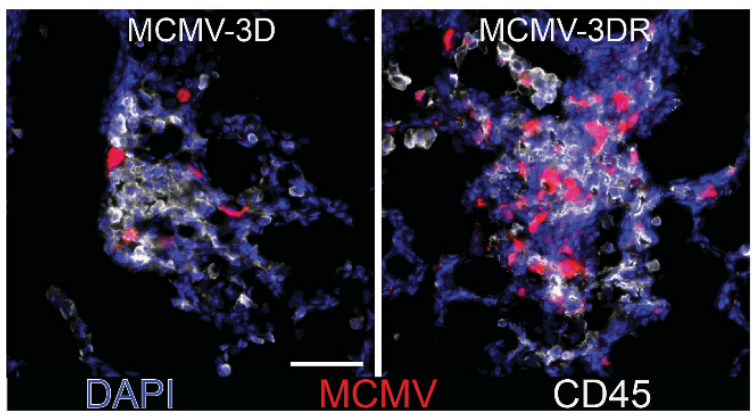

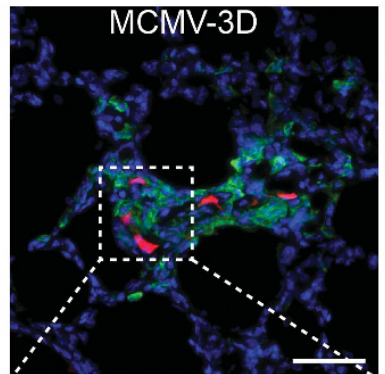
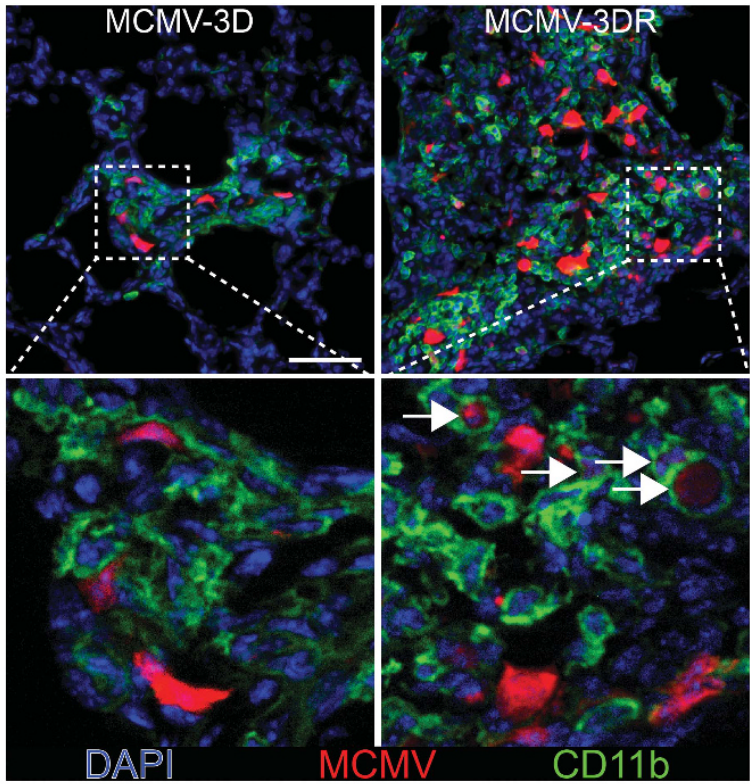

f

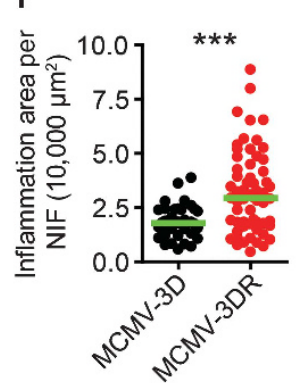

g

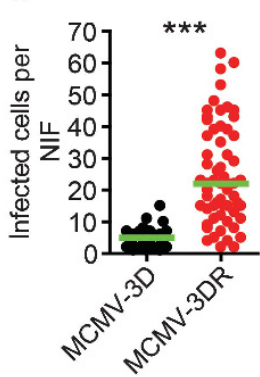

h

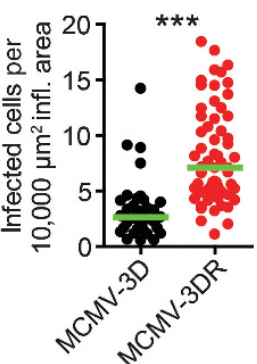

Figure 2 Viral infection with $\mathrm{Mck}^{+}$mouse cytomegalovirus (MCMV) is increased in nodular inflammatory foci (NIF) of the neonatal lung. $(\mathbf{a}-\mathbf{h})$ Neonatal mice were laryngopharyngeally infected with $5 \times 10^{4}$ plaque-forming units (PFU) MCMV mutants as indicated. Frozen lung sections were analyzed at 5 days post infection with antibody and 4,6-diamidino-2-phenylindole (DAPI) staining as indicated. (b and c) Frequency of NIFs per left lung lobe. (b) Frames highlight NIFs that are composed of infected cells and inflammatory infiltrate. (c) Quantitative analysis of NIF frequency per left lung lobe, two slides per animal were counted; lines indicate medians. (d and e) Higher magnification of representative NIFs from neonates infected with MCMV mutants as indicated. (e) Arrows indicate CD11 b ${ }^{+}$mCherry ${ }^{+}$cells. (f-h) Quantitative analysis of inflammation area per NIF and numbers of infected cells per NIF or per inflammation area; lines indicate medians. Data were obtained from four independent experiments with $n=7-8$ animals per group. (b) Bar $=500 \mu \mathrm{m}$ and (d and e) bar $=50 \mu \mathrm{m}$. MCK2, MCMV-encoded chemokine 2 . Statistical significance depicted as follows: ${ }^{* *} P<0.001$.

have previously reported that MCMV-3D primarily infects lung epithelial cells, mostly type 2 alveolar epithelial cells. ${ }^{9}$ Accordingly, we found all cells infected with MCMV-3D at $1 \mathrm{dpi}$ to be positioned in the lung epithelium of neonatal mice (Figure 3a,b). In contrast, approximately $60 \%$ of cells infected with MCMV-3DR were located inside the alveolar space (intraalveolar; Figure 3a,b). Next, we performed bronchoalveolar lavage (BAL) of MCMV-3D- or MCMV-3DR-infected neonatal mice. We could not isolate mCherry ${ }^{+}$cells from MCMV-3D-infected neonates by this method (Figure 3c, d). In contrast, a reasonable number of isolated cells were found to be mCherry ${ }^{+}$in MCMV-3DR-infected mice at 1 dpi (Figure 3c, d). Further analysis of the mCherry ${ }^{+}$cells isolated by BAL of MCMV-3DR-infected neonates identified them to express CD11b, F4/80, CD11c and Siglec-F (Figure 3d). Similarly, we found $\mathrm{CD} 11 \mathrm{c}^{+}$Siglec- $\mathrm{F}^{+}$alveolar macrophages in lungs of adult mice to be infected following intranasal infection with MCMV-3DR but not with MCMV-3D (Supplementary Figure $\mathbf{1 b}$ and $\mathbf{c})$. However, expression levels of CD11c and Siglec-F on MCMV-3DR-infected neonatal BAL cells were to some degree lower than in adult infected cells. Furthermore, lower forward and side scatter signals of infected cells isolated from neonates suggested that these cells exhibit less volume and lower granularity than those isolated from adults. Alveolar macrophages originate from fetal monocytes and gain surface expression of CD11c and Siglec-F at postnatal days $1-3 .{ }^{26}$ Accordingly, BAL cells found to be infected in 2-days-old neonatal mice showed intermediate expression of CD11c and Siglec-F and therefore seemed to be in a transitional state from premature to mature alveolar macrophages.

To validate our findings from the in vivo experiments, we isolated BAL cells from adult mice and infected these in vitro 


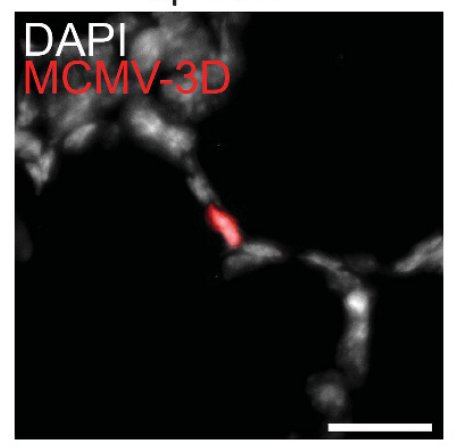

C

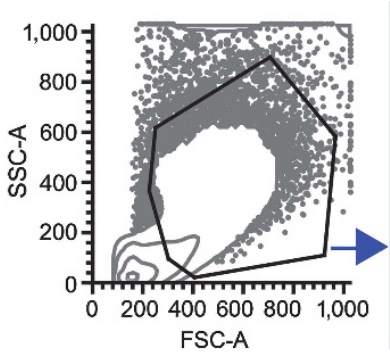

BAL cells - 1 dpi

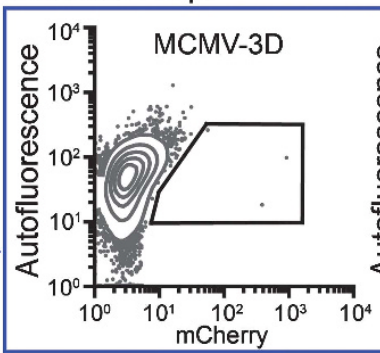

Intra-alveolar

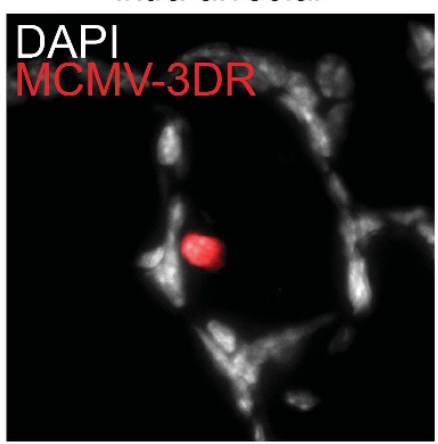

b

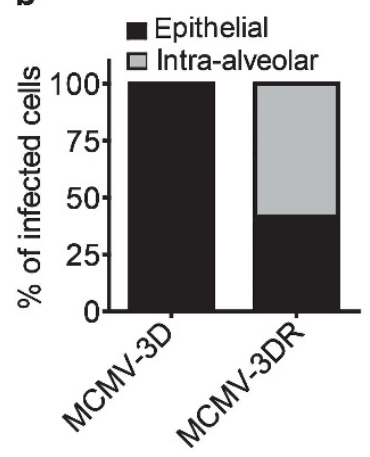

d
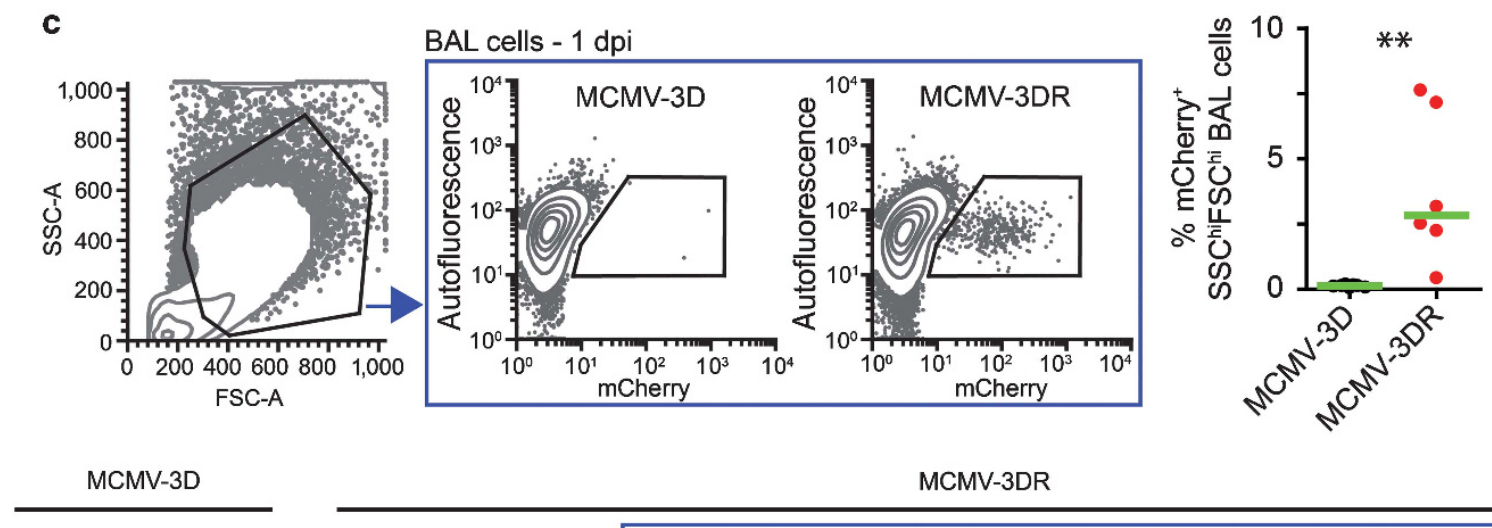

BAL cells - 1 dpi
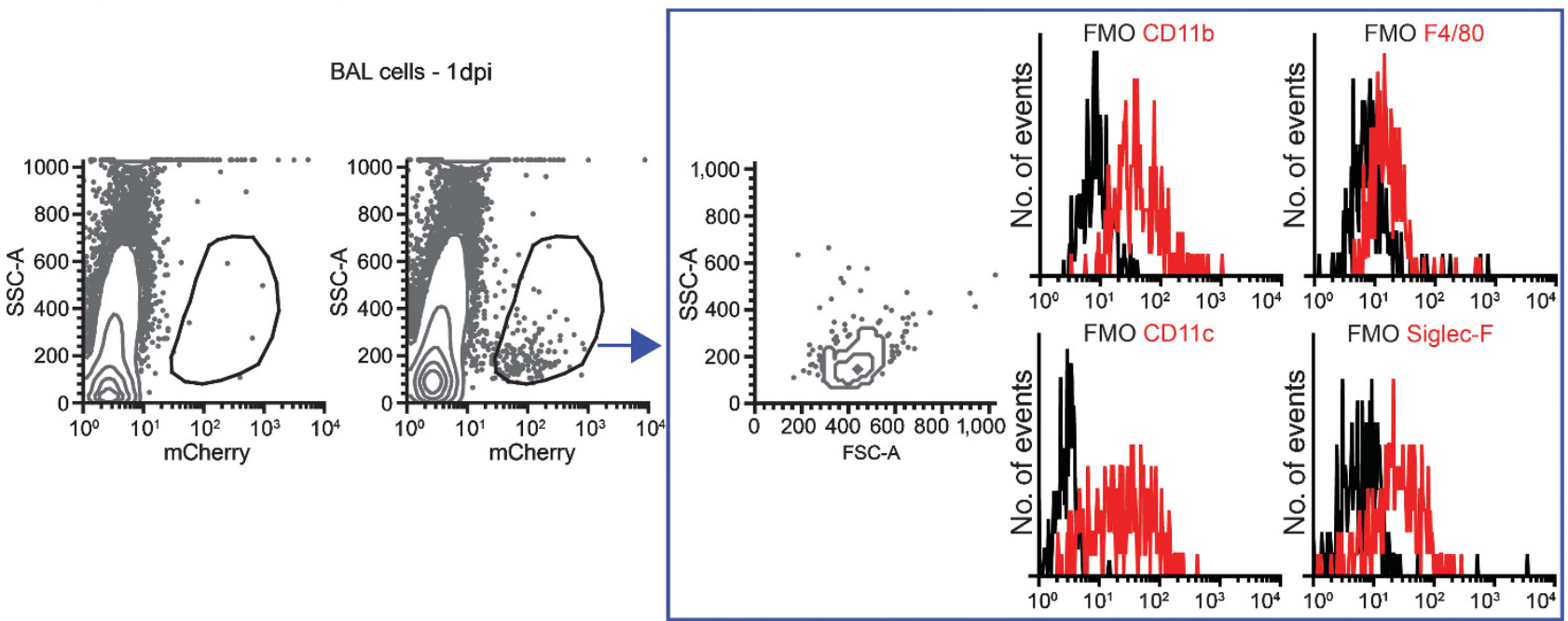

Figure 3 Mouse cytomegalovirus (MCMV)-3DR infects both neonatal alveolar macrophages and epithelial cells. (a-d) Neonatal mice were laryngopharyngeally infected with $5 \times 10^{4}$ plaque-forming units (PFU) MCMV mutants as indicated and analyzed at 1 day post infection (dpi). (a and b) Frozen sections of the lung were analyzed for the anatomical position of infected cells. Frequencies of infected lung epithelial cells or cells positioned in the alveolar space (intra-alveolar) were quantified. Data obtained from two animals per group with $n=59$ (MCMV-3D) and $n=43$ (MCMV-3DR) cells were analyzed. (c and d) Flow cytometry of bronchoalveolar lavage (BAL) cells from neonates infected with virus mutants as indicated.

(c) Frequency of mCherry ${ }^{+}$of SSC ${ }^{\text {hi }} \mathrm{FSC}^{\text {hi }}$ cells per BAL. One value represents one animal; lines indicate medians. (d) Surface marker expression of ex vivo antibody-stained $\mathrm{mCherry}^{+}$cells of MCMV-3DR-infected animals. Data were obtained from three independent experiments with $n=6-7$ animals per group. (a) Bar =20 $\mu \mathrm{m}$. DAPI, 4',6-diamidino-2-phenylindole; FMO, fluorescence minus one; FSC, forward scatter; SSC, side scatter. Statistical significance depicted as follows: ${ }^{* *} P<0.01$.

with increasing doses of MCMV-3D or MCMV-3DR. Alveolar macrophages, identified by their typical forward and side scatter signals and autofluorescence, were highly susceptible to MCMV-3DR but not to MCMV-3D infection (Figure 4a,b). Ten-fold higher multiplicities of infection were needed to infect at least some of the alveolar macrophages with MCMV-3D (unpublished data). As MCK2 shows homology to the CC chemokines CCL2, CCL3, CCL4, CCL5, and CCL11, ${ }^{14,17}$ we hypothesized that the difference in cell tropism of MCMV-3DR to alveolar macrophages might be mediated via interaction with a chemokine receptor. The aforementioned chemokines are supposed to bind to CCR1, CCR2, CCR3, and/or CCR5. ${ }^{27}$ 


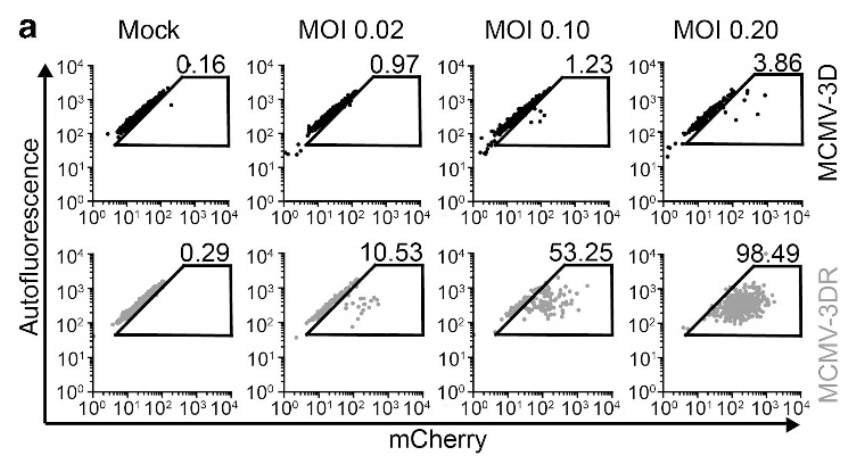

b

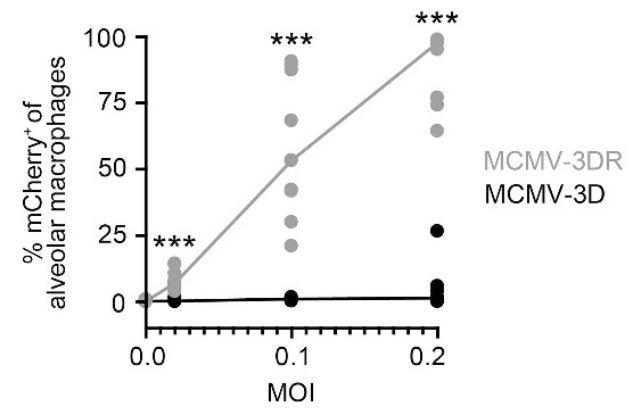

c

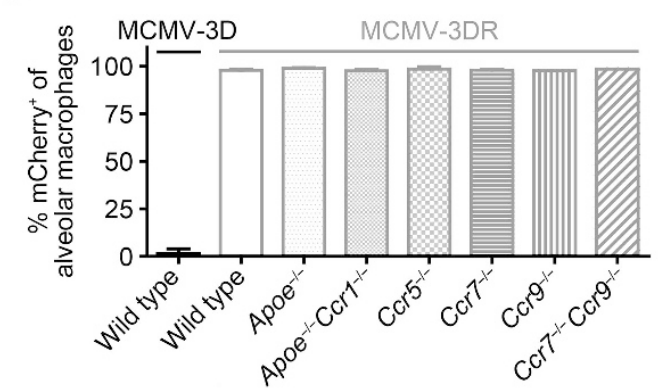

Figure 4 Ex vivo infection of adult alveolar macrophages. $(\mathrm{a}-\mathbf{c})$ Bronchoalveolar lavage (BAL) cells from adult mice were infected ex vivo and analyzed for mCherry expression $18 \mathrm{~h}$ after infection via flow cytometry. (a) Representative flow cytometry plots of alveolar macrophages infected with virus mutants and multiplicity of infections (MOIs) as indicated (viral titers were previously determined on murine embryonic fibroblasts (MEFs)). Numbers indicate percentage of infected cells. (b) Quantification of $\mathrm{mCherry}^{+}$alveolar macrophages per sample. Data were obtained from four experiments with a total of $n=7-13$ samples measured per group. (c) Alveolar macrophages isolated from various knockout animals were analyzed for susceptibility to MCMV-3DR infection. Data were obtained from one to two experiments with $n=3-13$ samples measured per group. Statistical significance depicted as follows: ${ }^{* * *} P<0.001$.

Although we failed to detect the expression of CCR1, CCR2, and CCR5 on infected BAL cells isolated from adult or neonatal mice by flow cytometry (Supplementary Figure 2a), alveolar macrophages have been predicted to express chemokine receptors including CCR1 and at low levels CCR2 and CCR5 on the transcript level (Immunological Genome Project; Heng and Painter ${ }^{28}$ ). Thus, we isolated alveolar macrophages from mice deficient for various CC chemokine receptors to investigate a potential role of these receptors during virus entry into the cell. Studying alveolar macrophages isolated from wild-type, CCR1-, CCR2-, CCR5-, CCR7-, or CCR9-deficient animals did not reveal any significant difference regarding susceptibility to MCMV-3DR infection, suggesting that infection of alveolar macrophages is independent of the receptors investigated (Figure $4 \mathrm{c}$ and Supplementary Figure 2b). Conclusively, the differences between the two MCMV mutants in replication within the neonatal lung at $5 \mathrm{dpi}$ were preceded by a significant difference in initial viral cell tropism.

\section{Depletion of alveolar macrophages (in vivo) reduces replication of MCMV-3DR in the neonatal lung}

We hypothesized that MCMV-3DR uses alveolar macrophages for a first round of replication to increase viral load. Thus, we laryngopharyngeally inoculated neonatal mice with either phosphate-buffered saline (PBS), liposomes (encapsome), or clodronate-liposomes (clodrosome). Clodronate-containing liposomes have been used in many studies to deplete alveolar macrophages and direct application into the lung is a well-established method. ${ }^{29,30}$ One day after this treatment, we infected neonatal mice with $5 \times 10^{4}$ PFU MCMV-3DR (Figure 5a). Clodrosomes reduced the total number of cells isolated from BAL (Figure 5b) and flow cytometry confirmed the reduction of $\mathrm{CD}_{11 \mathrm{c}^{+}}$Siglec-F ${ }^{+}$cells-indicating partial depletion of the MCMV-3DR-susceptible cells (Supplementary Figure 3a-e). No difference in viral activity was observed at 1 dpi between clodrosome- and encapsome-treated neonates, but it seemed that liposomes (compared to PBS) to some degree interfered with infection (Figure 5c). Strikingly, at $5 \mathrm{dpi}$ luciferase activity was significantly lower in the lungs, liver, and brain of clodrosome-treated animals when compared to the encapsome group (Figure 5d). Accordingly, we found less NIFs in the animals depleted of alveolar macrophages (Figure 5e,f) and high magnification analysis of NIFs revealed significant differences in virus replication between the two groups (Figure 5g). Although depletion of alveolar macrophages reduced the size of NIFs only marginally (Figure $5 \mathbf{h}$ ), the number of infected cells per NIF and per inflammation area was considerably decreased (Figure 5i,j). Importantly, when alveolar macrophages were depleted from neonates before MCMV-3D infection, viral activity at 5 dpi was comparable with that of mice receiving PBS or encapsome (Supplementary Figure 3f). These data indicate that the increased numbers of NIFs as well as the augmented local virus replication within NIFs observed after MCMV-3DR infection can be diminished by depletion of alveolar macrophages in the neonatal lung.

\section{DISCUSSION}

Approximately $1 \%$ of newborns are infected with HCMV at birth, but only a fraction of them show signs of viral disease. ${ }^{4}$ The occurrence of clinical symptoms depends on the host's condition, reflected by the observation of preterm low birth weight infants who are especially prone to suffer from HCMV infection. ${ }^{31-33}$ However, it is likely that polymorphism in viral genes also account for the genesis of CMV disease. ${ }^{34-36}$ In this study, we observed how a single-nucleotide deletion in the 
a

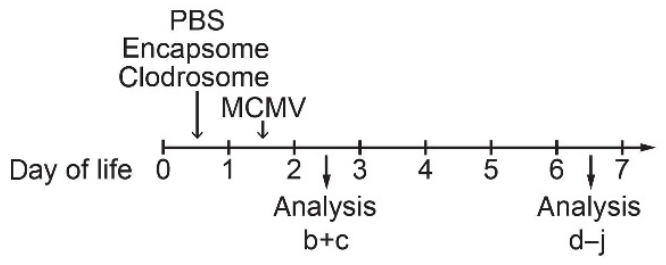

b

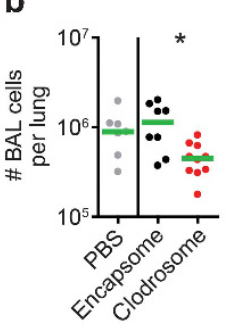

C

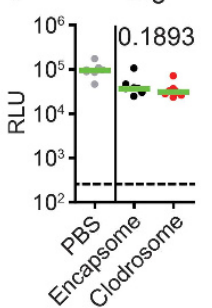

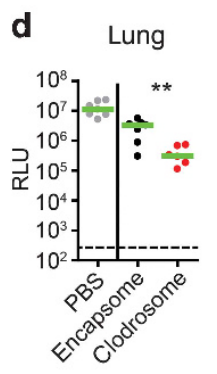

e

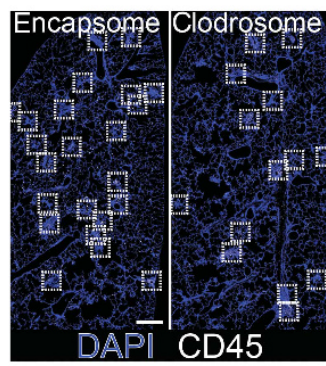

Liver

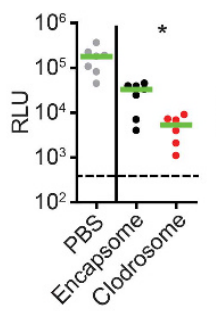

f

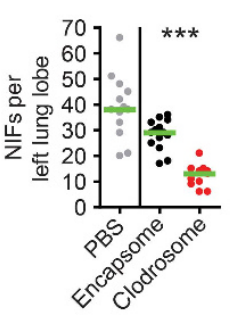

Brain

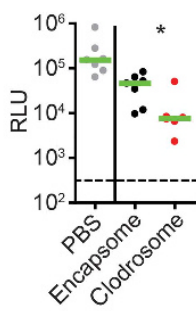

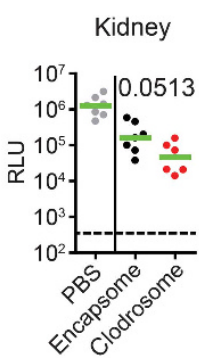
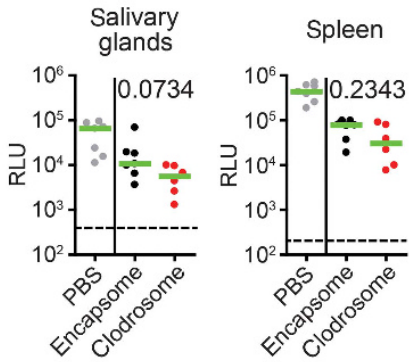

g

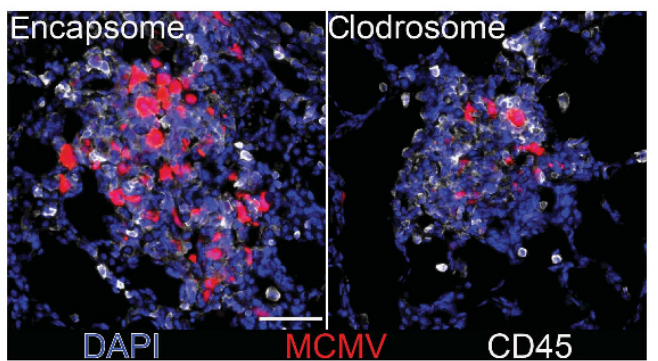

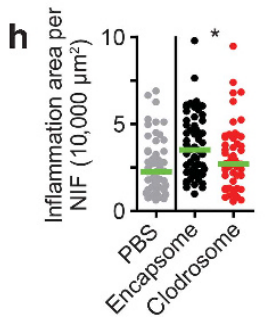
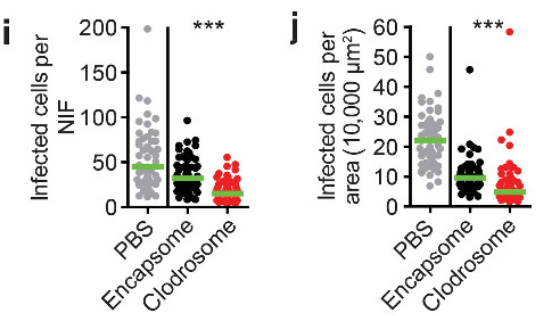

Figure 5 Depletion of alveolar macrophages reduces mouse cytomegalovirus (MCMV)-3DR infection in the neonatal lung. (a-j) Phosphate-buffered saline (PBS), encapsome, or clodrosome was laryngopharyngeally inoculated into neonatal mice. One day later, animals were infected with $5 \times 10^{4}$ plaque-forming units (PFU) of MCMV-3DR as indicated and analyzed at (b and c) 1 day post infection (dpi) or (d-j) 5 dpi. (b) Clodrosome-mediated cell depletion was measured by counting of bronchoalveolar lavage (BAL) cells per animal. One value represents one animal. Data were obtained from three independent experiments with $n=7-10$ animals per group. (c and d) Luciferase activity in neonatal organs determined at (c) 1 dpi and (d) 5 dpi. Data were obtained from two to three independent experiments with $n=7-8$ animals per group; lines indicate medians and dashed lines indicate detection limits. (e-j) Histological analysis of neonatal left lung lobes at 5 dpi with MCMV-3DR. (e and $\mathbf{f}$ ) Number of nodular inflammatory foci (NIFs) per left lung lobe at 5 dpi. (e) NIFs are highlighted by frames. (f) Quantitative analysis of NIF numbers per left lung lobe, two slides per animal were counted; lines indicate medians. (g) Higher magnification of representative NIFs from neonates infected with MCMV-3DR. (h-j) Quantitative analysis of inflammation area per NIF and number of infected cells per NIF or per inflammation area; lines indicate medians. $(\mathbf{d}-\mathbf{j})$ Data were obtained from three independent experiments with $n=6-7$ animals per group. Non-parametric $t$-tests between encapsome- and clodrosome-treated groups. (e) $\mathrm{Bar}=500 \mu \mathrm{m}$ and $(\mathbf{g})$ bar $=50 \mu \mathrm{m}$. Statistical significance depicted as follows: ${ }^{*} P<0.05 ;{ }^{\star \star} P<0.01 ;$ and ${ }^{\star \star \star} P<0.001$.

MCMV genome has a marked effect on virus biology and subsequently on the health status of the neonatal host. Reinsertion of a single base pair into MCMV-3D profoundly modified the primary cell tropism of the virus in the lung. Whereas both mutants, MCMV-3D and MCMV-3DR, infected epithelial cells, MCMV-3DR also showed a pronounced tropism to alveolar macrophages. The base pair deletion in the $m 129$ of MCMV-3D is predicted to lead to a frameshift and therefore to a truncated MCK2 protein. ${ }^{19}$ Thus, enabling accurate translation of the Mck2 ORF in the MCMV-3DR mutant represents a gain-of-function mutation with substantial in vivo consequences regarding pathogenicity and virus activity.

The role of $M c k 2$ in virus replication has been studied before in adolescent or adult mice. Increased viral titers in the salivary glands after intraperitoneal or subcutaneous injection of 
$M c k 2^{+}$virus have been reported, ${ }^{16,18,19}$ and elevated viral loads in the spleen with $\mathrm{Mck2}^{+}$virus were observed. ${ }^{16,20}$ However, using these routes of infection 3- to 8-week-old BALB/c mice showed no other obvious differences in viral replication in organs other than the salivary glands. ${ }^{16,18,19}$ Similarly, in the present study, we found virus activity in salivary glands of adult mice infected with MCMV-3DR to be higher than in MCMV3D-infected animals. In contrast, applying lung infection to neonatal mice, we observed the $M c k 2^{+}$MCMV-3DR to replicate significantly higher than the $M c k 2^{-}$MCMV-3D in all organs tested. In addition, the majority of neonates infected with MCMV-3DR suffered from viral disease and had to be killed. Neonatal mice are in general more susceptible to MCMV infection than adults. ${ }^{5,6,9}$ Thus, independent of Mck2, multiorgan infection following administration of MCMV into the lung has been observed in neonates but not in adults. ${ }^{9}$ Furthermore, in neonates replication of MCMV-3DR was significantly higher in the lung and subsequently in all organs when compared to MCMV-3D. This phenomenon was accompanied by a significant difference in initial viral cell tropism in the lung toward alveolar macrophages.

Alveolar macrophages are part of the first-line defense of the lung mucosa. In addition to clearance of surfactant, they take up inhaled particles to prevent systemic invasion of microbes or toxic substances. These cells are thought to possess rather suppressive than proinflammatory features to avoid overwhelming inflammatory reactions and dysfunction of gas exchange. ${ }^{37}$ Here, we identified these cells as a target of CMV infection. Infection of alveolar macrophages with MCMV-3DR amplified the formation of NIFs and promoted replication of the virus within these structures. Furthermore, depletion of alveolar macrophages before infection with MCMV-3DR decreased subsequent viral loads in the lung and could protect to some degree from systemic infection. Obviously, MCMV takes advantage of the accessibility of alveolar macrophages that are present in substantial numbers in the lower respiratory tract. Thus, MCMV might exploit these cells to multiply initial virus replication in vivo. This could explain-at least in part-why MCMV-3DR shows higher pathogenicity in neonates.

Macrophages have been proposed as target cells for MCMV infection. ${ }^{38-40}$ In a recent report, the role of $M c k 2$ in infection of peritoneal macrophages was addressed. ${ }^{41}$ Interestingly, these authors found $\mathrm{Mck2}^{+}$virus to infect approximately twofold more $\mathrm{CD} 11 \mathrm{~b}^{+} \mathrm{F} 4 / 80^{+}$macrophages than an $\mathrm{Mck} 2^{-} \mathrm{MCMV}$ mutant. However, even the $M c k 2^{-}$mutant was able to infect up to $1 \%$ of these peritoneal macrophages. In the present study, we found up to $7.5 \%$ of BAL cells to be infected with MCMV-3DR, whereas BAL cells from MCMV-3D-infected neonates were not infected. Taken together, these studies reveal remarkable differences in the first cell type to be infected after intraperitoneal or laryngopharyngeal infection. Alveolar macrophages are phenotypically distinct from peritoneal macrophages ${ }^{42}$ and seem to be susceptible to MCMV-3DR infection but not to MCMV-3D. This is supported by our in vitro studies of adult alveolar macrophages where 10 -fold more virus was needed to infect these cells with MCMV-3D. Thus, the route of virus application and the initially infected cell type in the exposed organ has important effects on the subsequent course of MCMV infection.

In the present study, we also addressed the role of CC chemokine receptors in MCMV-3DR infection of alveolar macrophages. We aimed to investigate receptors that were described to interact with ligands that show sequence homology to MCK2 and have been predicted to be expressed in alveolar macrophages. From the data obtained, it seemed as if CCR1, CCR2, CCR5, CCR7, and CCR9 were not essential for MCMV3DR infection of alveolar macrophages. However, it has to be considered that MCK2 may use more than only one receptor and the CCRs analyzed in this study may substitute for each other to allow the virus to infect the cell. Furthermore, a predicted surface membrane expression of CCR1, 2, and 5 could not be confirmed on infected alveolar macrophages by flow cytometry. Thus, the role of CC chemokine receptors in infection of macrophages needs further investigation.

It has recently been proposed that the CC chemokine homolog MCK2 forms a complex with the MCMV glycoproteins $\mathrm{gH}$ and $\mathrm{gL}$ to promote infection of peritoneal and liver macrophages in vivo. ${ }^{41}$ Interestingly, CC chemokine homolog encoding sequences have been described in CMVs of humans, chimpanzees, simians, rats, and guinea-pigs. ${ }^{43-46}$ Thus, studying Mck2 in the mouse model likely reveals features of MCMV that might be applicable to CMVs of other species. The protein encoded by the corresponding HCMV gene UL128 has been shown to form a pentameric complex together with gH, gL, pUL130, and pUL131, ${ }^{47-49}$ which has been implicated in virus tropism. The pentameric complex is required for entry of HCMV strains into various cell types, including epithelial and endothelial cells, polymorphonuclear leukocytes, and dendritic cells, ${ }^{50}$ whereas HCMV mutants with a mutation in UL128, UL130, or UL131A grow unaffected in human embryonic lung fibroblasts. ${ }^{44,51}$ Furthermore, the HCMV gH/gL/UL131-128 complex has been shown to be essential for productive internalization of virions into monocytes. ${ }^{52,53}$ Based on these data, the UL128-131 proteins were proposed to have a central role in dissemination of the virus using monocytes as vehicles. ${ }^{50,54}$ Similarly, blood monocytes are implicated in viral dissemination in the model of MCMV and Mck2 is supposed to promote this process. ${ }^{17,18,21,55}$ In line with these observations are reports from CMVs of rat and guineapig. ${ }^{45,46,56}$ Conclusively, the MCK2 protein and its homologs in CMVs of various hosts are involved in viral cell tropism, viral dissemination, and hence in viral pathogenicity.

In summary, we investigated the role of Mck2 in MCMV infection of the neonatal respiratory tract. We found that reconstitution of the Mck2 ORF had a tremendous impact on the pathogen's virulence in neonatal mice. $M c k 2$ is needed to infect alveolar macrophages in vivo and allows MCMV to use this cell type to increase viral loads in the lung mucosa. It further promotes viral dissemination, possibly monocyte-associated, and leads to disease of the neonatal host. Depletion of this first target cell in vivo diminished subsequent viral replication 
in the lung and other organs. We propose that MCMV has evolved an Mck2-dependent mechanism to use a specific host cell, namely the alveolar macrophage, to amplify infection in the lung mucosa.

\section{METHODS}

Animals. All mice were on a C57BL/6 background, bred at the central animal facility of Hannover Medical School (Hannover, Germany) under specific pathogen-free conditions and/or purchased from Charles River Laboratories (Sulzfeld, Germany). Apoe ${ }^{-1-}$, Apoe $^{-1-} \mathrm{Ccr}^{-1-}, \mathrm{Ccr} 2^{-1-}, \mathrm{Ccr} 5^{-1-}, \mathrm{Ccr} 7^{-1-}$, and $C \mathrm{Cr} 9^{-1-}$ have been described; $C \mathrm{cr} 7^{-1-} \mathrm{C} c r 9^{-1-}$ mice were backcrossed to the C57BL/6 background for at least eight generations.

Ethics statement. All animal experiments were performed according to the recommendations and guidelines of the Federation of European Laboratory Animal Science Associations (FELASA) and Society of Laboratory Animals (GV-SOLAS) and approved by the institutional review board and the Niedersächsische Landesamt für Verbraucherschutz und Lebensmittelsicherheit (AZ33.9-42502-04$10 / 0225,-12 / 0921$, and $-13 / 1255)$. Termination criteria for infection experiments of neonatal mice (animals fulfilling one of the following criteria were killed) were as follows: (1) no distinct movements, even after manipulation of body position; (2) persistent cyanosis or paleness; (3) anorexia, significantly decreased body weight gain and obviously decreased skin turgor.

Viruses and infections. MCMV-3D mutant has been described previously ${ }^{25}$ and used for the repair of the m129 ORF using the en passant mutagenesis protocol as described. ${ }^{19}$ Correct insertion of the missing nucleotide was verified by sequence analysis. Both mutants encode Gaussia luciferase and mCherry, and carry an additional sequence within the m164 ORF encoding the SIINFEKL peptide. Preparation of MCMV stocks and plaque assays were performed as described previously. ${ }^{25}$ Neonatal mice were infected within their first $24 \mathrm{~h}$ of life if not indicated differently. Lung infections were performed by inoculations of a volume of $10 \mu \mathrm{l}$ by probing the laryngopharynx with a pipette and extension of the neck (laryngopharyngeal infection). Adult mice, 6-8 week olds, were anesthetized (ketamine and xylazine) and $40 \mu \mathrm{l}$ of virus solution was applied to the nostrils for intranasal infection. All experiments for comparative analysis of MCMV-3D and MCMV-3DR have been performed in parallel with simultaneously titrated virus stocks.

Alveolar macrophage depletion and isolation of bronchoalveolar lavage cells. PBS or liposomes of the 'Standard Macrophage Depletion Kit' (Encapsula NanoSciences LLC, Nashville, TN) were laryngopharyngeally applied $(10 \mu \mathrm{l}$ per animal) into $<24 \mathrm{~h}$ old neonates and infected with MCMV the subsequent day. Animals were killed one day after infection and the right heart ventricle was gently perfused with cold PBS to remove blood cells from the lung. The surface of the explanted lung was flushed in PBS to remove pleural cells and then gently filled with $300 \mu \mathrm{l}$ cold PBS intratracheally (this procedure was repeated two times). Returning fluid was collected for the generation of cell suspensions. After antibody staining, cells were processed with LSRII Cytometer (BD Biosciences, San Jose, CA) and data were analyzed with BD FACSDiva Software (6.1.3; BD Biosciences) or WinList 6.0 software (Verity Software House, Topsham, ME).

Ex vivo infection of adult BAL cells and quantification of infected alveolar macrophages. To isolate BAL cells from adult mice, lungs were filled in situ with $1000 \mu \mathrm{l}$ cold PBS, the returning fluid was collected (this procedure was repeated two times). Cells were counted (Vi-Cell; Beckman Coulter, Krefeld, Germany), plated on 96-well plates in RPMI media, and subsequently infected with different multiplicities of infection of MCMV mutants. Cells were isolated $18 \mathrm{~h}$ after infection using 0.25\% trypsin (Biochrom AG, Berlin, Germany) for the detachment of macrophages. Flow cytometry was performed and alveolar macrophages were identified by forward and side scatter position and autofluorescence.

Histology and antibodies. For histological analysis, organs were fixed in $2 \%$ paraformaldehyde and $30 \%$ sucrose for $30 \mathrm{~min}$ and embedded in OCT compound (Tissue-Tek; Sakura, Leiden, The Netherlands). Seven-micrometer-thick organ slices were stained after appropriate blocking with depicted antibodies. Images were taken with an AxioCam MRm camera (Carl Zeiss, Jena, Germany) attached to Axiovert 200M fluorescence microscope (Carl Zeiss) with PlanApochromat objectives $\times 10 / 0.45, \times 20 / 0.75$, and $\times 40 / 0.95$ (magnification/numerical aperture) and processed with AxioVision 4.8 software (Carl Zeiss). In some cases (magnification images in Figure 2e), structured illumination microscopy was performed with the ApoTome (Carl Zeiss). All images were processed with Microsoft Office Picture Manager. The following antibodies (clones) were used after adequate blocking of Fc receptors: CD11b-eFluor450 (M1/70), CD11beFluor660 (M1/70), CD11c-PE/Cy7 (N418), CD45-APC (30-F11), CCR1-PE (643854), CCR2-PE (475301), CCR5-PE (C34-3448), F4/80eFluor780 (BM8), F4/80-PE (BM8), isotype IgG2c-PE (A23-1), isotype IgG2b-PE (eB149/10H5), and Siglec-F-AlexaFluor647(E50-2440).

Luciferase measurements. Organ preparations were performed after perfusion of supplying blood vessels with PBS. Explanted organs were kept in PBS, homogenized with TissueLyser II (Qiagen, Venlo, The Netherlands), centrifuged, and supernatants were measured for luciferase expression after the addition of native Coelenterazine (Synchem, Felsberg, Germany) with Lumat LB 9507 (Berthold Technologies, Bad Wildbad, Germany). For the lung, salivary glands and liver, 1:10 dilutions were performed for measurements.

Quantification of infected cells. Histology sections of the left lung lobe were analyzed. Area of inflammation was determined by manual measurement of $\mathrm{CD} 45^{+}$stain signal using AxioVision 4.8 software.

Statistical analysis. Statistical analysis was performed with Prism 4 (GraphPad, San Diego, CA). Unpaired non-parametric $t$-test was used for comparison of two groups. Statistical significance was depicted as follows: NS, $P>0.05$; ${ }^{\star} P<0.05$; ${ }^{* *} P<0.01$; and ${ }^{* * *} P<0.001$.

SUPPLEMENTARY MATERIAL is linked to the online version of the paper at http://www.nature.com/mi

\section{ACKNOWLEDGMENTS}

We thank Mathias Herberg for excellent animal care. We also thank Denise Hilfiker-Kleiner for kindly providing $\mathrm{Ccr} 2^{-1-}$ mice, Christian Weber for kindly providing Apoe ${ }^{-1-} \mathrm{CCr}^{-1-}$ mice, and Gregor Theilmeier for kindly providing Apoe ${ }^{-1-}$ mice. This work benefitted from data assembled by the ImmGen consortium. This work was supported by Deutsche Forschungsgemeinschaft (DFG) grant SFB900-B1 to R.F. and M.M., the Hannover Biomedical Research School (HBRS), and the Center for Infection Biology (ZIB). The funders had no role in study design, data collection and analysis, decision to publish, or preparation of the manuscript.

\section{DISCLOSURE}

The authors declared no conflict of interest.

(c) 2015 Society for Mucosal Immunology

\section{REFERENCES}

1. Virgin, H.W. In vivo veritas: pathogenesis of infection as it actually happens. Nat. Immunol. 8, 1143-1147 (2007).

2. Scalzo, A.A., Corbett, A.J., Rawlinson, W.D., Scott, G.M. \& Degli-Esposti, M.A. The interplay between host and viral factors in shaping the outcome of cytomegalovirus infection. Immunol. Cell Biol. 85, 46-54 (2007). 
3. Reddehase, M.J. Antigens and immunoevasins: opponents in cytomegalovirus immune surveillance. Nat. Rev. Immunol. 2, 831-844 (2002).

4. Mocarski, E.S., Shenk, T. \& Pass, R. Cytomegalovirus. In Fields Virology, 5th edn (Knipe, D.M. \& Howley, P.M., eds) 2702-2772 (Lippincott Williams \& Wilkins, Philadelphia, PA, 2007).

5. Selgrade, M.K. \& Osborn, J.E. Role of macrophages in resistance to murine cytomegalovirus. Infect. Immun. 10, 1383-1390 (1974).

6. Fitzgerald, N.A., Papadimitriou, J.M. \& Shellam, G.R. Cytomegalovirusinduced pneumonitis and myocarditis in newborn mice. A model for perinatal human cytomegalovirus infection. Arch. Virol. 115, 75-88 (1990).

7. Trgovcich, J., Pernak-Pugel, E., Tomac, J., Koszinowski, U.H. \& Jonjic, S. Pathogenesis of murine cytomegalovirus infection in neonatal mice. In CMV-Related Immunopathology Monogr Virol, (Scholz, M., Rabenau, H.F., Doerr, H.W. \& Cinatl, J. Jr. eds) (Karger, Basel, Switzerland, 1998).

8. Krmpotic, A., Bubic, I., Polic, B., Lucin, P. \& Jonjic, S. Pathogenesis of murine cytomegalovirus infection. Microbes Infect. 5, 1263-1277 (2003).

9. Stahl, F.R. et al. Nodular inflammatory foci are sites of T cell priming and control of murine cytomegalovirus infection in the neonatal lung. PLoS Pathogen. 9, e1003828 (2013).

10. Messerle, M., Crnkovic, I., Hammerschmidt, W., Ziegler, H. \& Koszinowski, U.H. Cloning and mutagenesis of a herpesvirus genome as an infectious bacterial artificial chromosome. Proc. Natl. Acad. Sci. USA 94, 14759-14763 (1997).

11. Ruzsics, Z., Borst, E.M., Bosse, J.B., Brune, W. \& Messerle, M. Manipulating cytomegalovirus genomes by BAC mutagenesis: strategies and applications. In Cytomegalovirus: From Molecular Pathogenesis to Intervention, Voll (Reddehase, M.J., ed) 37-57 (Caister Academic Press, Hethersett, Norwich, UK, 2013).

12. Lemmermann, N.A. et al. Murine cytomegalovirus immune evasion proteins operative in the $\mathrm{MHC}$ class I pathway of antigen processing and presentation: state of knowledge, revisions, and questions. Med. Microbiol. Immunol. 201, 497-512.

13. Jonjic, S., Babic, M., Polic, B. \& Krmpotic, A. Immune evasion of natural killer cells by viruses. Curr. Opin. Immunol. 20, 30-38 (2008).

14. MacDonald, M.R., Li, X.Y. \& Virgin, H.W.t. Late expression of a beta chemokine homolog by murine cytomegalovirus. J. Virol. 71, 1671-1678 (1997).

15. MacDonald, M.R., Burney, M.W., Resnick, S.B. \& Virgin, H.I. Spliced mRNA encoding the murine cytomegalovirus chemokine homolog predicts a beta chemokine of novel structure. J. Virol. 73, 3682-3691 (1999).

16. Fleming, P. et al. The murine cytomegalovirus chemokine homolog, m131/ 129 , is a determinant of viral pathogenicity. J. Virol. 73, 6800-6809 (1999).

17. Saederup, N., Lin, Y.C., Dairaghi, D.J., Schall, T.J. \& Mocarski, E.S. Cytomegalovirus-encoded beta chemokine promotes monocyte-associated viremia in the host. Proc. Natl Acad. Sci. USA 96, 10881-10886 (1999).

18. Saederup, N., Aguirre, S.A., Sparer, T.E., Bouley, D.M. \& Mocarski, E.S. Murine cytomegalovirus CC chemokine homolog MCK-2 (m131-129) is a determinant of dissemination that increases inflammation at initial sites of infection. J. Virol. 75, 9966-9976 (2001).

19. Jordan, S. et al. Virus progeny of murine cytomegalovirus bacterial artificial chromosome PSM3fr show reduced growth in salivary glands due to a fixed mutation of MCK-2. J. Virol. 85, 10346-10353 (2011).

20. Wikstrom, M.E., Fleming, P., Comerford, I., McColl, S.R., Andoniou, C.E. \& Degli-Esposti, M.A. A chemokine-like viral protein enhances alpha interferon production by plasmacytoid dendritic cells but delays CD8 + Tcell activation and impairs viral clearance. J. Virol. 87, 7911-7920 (2013).

21. Noda, S. et al. Cytomegalovirus MCK-2 controls mobilization and recruitment of myeloid progenitor cells to facilitate dissemination. Blood 107, 30-38 (2006).

22. Wikstrom, M.E. et al. The early monocytic response to cytomegalovirus infection is MyD88-dependent but occurs independently of common inflammatory cytokine signals. Eur. J. Immunol. 44, 409-419 (2014).

23. Daley-Bauer, L.P., Wynn, G.M. \& Mocarski, E.S. Cytomegalovirus impairs antiviral CD8 + T cell immunity by recruiting inflammatory monocytes. Immunity 37, 122-133 (2012).

24. Wagner, M., Jonjic, S., Koszinowski, U.H. \& Messerle, M. Systematic excision of vector sequences from the BAC-cloned herpesvirus genome during virus reconstitution. J. Virol. 73, 7056-7060 (1999).
25. Marquardt, A. et al. Single cell detection of latent cytomegalovirus reactivation in host tissue. J. Gen. Virol. 92 (Part 6), 1279-1291 (2011).

26. Guilliams, M. et al. Alveolar macrophages develop from fetal monocytes that differentiate into long-lived cells in the first week of life via GM-CSF. J. Exp. Med. 210, 1977-1992 (2013).

27. Zlotnik, A. \& Yoshie, O. The chemokine superfamily revisited. Immunity 36, 705-716 (2012).

28. Heng, T.S. \& Painter, M.W. The Immunological Genome Project: networks of gene expression in immune cells. Nat. Immunol. 9, 1091-1094 (2008).

29. Thepen, T., Van Rooijen, N. \& Kraal, G. Alveolar macrophage elimination in vivo is associated with an increase in pulmonary immune response in mice. J. Exp. Med. 170, 499-509 (1989).

30. Chow, A., Brown, B.D. \& Merad, M. Studying the mononuclear phagocyte system in the molecular age. Nat. Rev. Immunol. 11, 788-798 (2011).

31. Lombardi, G., Garofoli, F., Manzoni, P. \& Stronati, M. Breast milk-acquired cytomegalovirus infection in very low birth weight infants. J. Matern. Fetal Neonatal Med. 25 (Suppl 3), 57-62 (2012).

32. Coclite, E., Di Natale, C. \& Nigro, G. Congenital and perinatal cytomegalovirus lung infection. J. Matern. Fetal Neonatal Med. 26, 1671-1675 (2013).

33. Kenneson, A. \& Cannon, M.J. Review and meta-analysis of the epidemiology of congenital cytomegalovirus (CMV) infection. Rev. Med. Virol. 17, 253-276 (2007).

34. Pignatelli, S., Dal Monte, P., Rossini, G. \& Landini, M.P. Genetic polymorphisms among human cytomegalovirus (HCMV) wild-type strains. Rev. Med. Virol. 14, 383-410 (2004).

35. Smith, L.M., Shellam, G.R. \& Redwood, A.J. Genes of murine cytomegalovirus exist as a number of distinct genotypes. Virology 352, 450-465 (2006).

36. Renzette, N., Bhattacharjee, B., Jensen, J.D., Gibson, L. \& Kowalik, T.F. Extensive genome-wide variability of human cytomegalovirus in congenitally infected infants. PLoS Pathogen. 7, e1001344 (2011).

37. Lambrecht, B.N. Alveolar macrophage in the driver's seat. Immunity $\mathbf{2 4}$, 366-368 (2006).

38. Mims, C.A. \& Gould, J. The role of macrophages in mice infected with murine cytomegalovirus. J. Gen. Virol. 41, 143-153 (1978).

39. Brautigam, A.R., Dutko, F.J., Olding, L.B. \& Oldstone, M.B. Pathogenesis of murine cytomegalovirus infection: the macrophage as a permissive cell for cytomegalovirus infection, replication and latency. J. Gen. Virol. 44, 349-359 (1979).

40. Shanley, J.D. \& Pesanti, E.L. Murine peritoneal macrophages support murine cytomegalovirus replication. Infect. Immun. 41, 1352-1359 (1983).

41. Wagner, F.M. et al. The viral chemokine MCK-2 of murine cytomegalovirus promotes infection as part of a gH/gL/MCK-2 complex. PLoS Pathogen. $\mathbf{9}$, e1003493 (2013).

42. Davies, L.C., Jenkins, S.J., Allen, J.E. \& Taylor, P.R. Tissue-resident macrophages. Nat. Immunol. 14, 986-995 (2013).

43. Akter, P. et al. Two novel spliced genes in human cytomegalovirus. J. Gen. Virol. 84 (Part 5), 1117-1122 (2003).

44. Hahn, G. et al. Human cytomegalovirus UL131-128 genes are indispensable for virus growth in endothelial cells and virus transfer to leukocytes. J. Virol. 78, 10023-10033 (2004).

45. Kaptein, S.J. et al. The r131 gene of rat cytomegalovirus encodes a proinflammatory CC chemokine homolog which is essential for the production of infectious virus in the salivary glands. Virus Genes 29, 43-61 (2004).

46. Auerbach, M. et al. Characterization of the guinea pig CMV gH/gL/GP129/ GP131/GP133 complex in infection and spread. Virology 441, 75-84.

47. Wang, D. \& Shenk, T. Human cytomegalovirus virion protein complex required for epithelial and endothelial cell tropism. Proc. Natl Acad. Sci. USA 102, 18153-18158 (2005).

48. Adler, B., Scrivano, L., Ruzcics, Z., Rupp, B., Sinzger, C. \& Koszinowski, U. Role of human cytomegalovirus UL131A in cell type-specific virus entry and release. J. Gen. Virol. 87 (Part 9), 2451-2460 (2006).

49. Ryckman, B.J. et al. Characterization of the human cytomegalovirus $\mathrm{gH} /$ gL/UL128-131 complex that mediates entry into epithelial and endothelial cells. J. Virol. 82, 60-70 (2008).

50. Revello, M.G. \& Gerna, G. Human cytomegalovirus tropism for endothelial/ epithelial cells: scientific background and clinical implications. Rev. Med. Virol. 20, 136-155 (2010). 
51. Gerna, G. et al. Dendritic-cell infection by human cytomegalovirus is restricted to strains carrying functional UL131-128 genes and mediates efficient viral antigen presentation to CD8 + Tcells. J. Gen. Virol. 86 (Part 2), 275-284 (2005).

52. Straschewski, S., Patrone, M., Walther, P., Gallina, A., Mertens, T. \& Frascaroli, G. Protein pUL128 of human cytomegalovirus is necessary for monocyte infection and blocking of migration. J. Virol. 85, 5150-5158 (2011).

53. Nogalski, M.T., Chan, G.C., Stevenson, E.V., Collins-McMillen, D.K. \& Yurochko, A.D. The HCMV gH/gL/UL128-131 complex triggers the specific cellular activation required for efficient viral internalization into target monocytes. PLoS Pathogen. 9, e1003463 (2013).
54. Smith, M.S., Bentz, G.L., Alexander, J.S. \& Yurochko, A.D. Human cytomegalovirus induces monocyte differentiation and migration as a strategy for dissemination and persistence. J. Virol. 78, 4444-4453 (2004).

55. Stoddart, C.A., Cardin, R.D., Boname, J.M., Manning, W.C., Abenes, G.B. \& Mocarski, E.S. Peripheral blood mononuclear phagocytes mediate dissemination of murine cytomegalovirus. J. Virol. 68, 6243-6253 (1994).

56. Nozawa, N. et al. Identification of a $1.6 \mathrm{~kb}$ genome locus of guinea pig cytomegalovirus required for efficient viral growth in animals but not in cell culture. Virology 379, 45-54 (2008). 